\title{
The Genetics of Common Epilepsy Disorders: Lessons Learned from the Channelopathy Era
}

\author{
Ryan L. Subaran · David A. Greenberg
}

Published online: 5 August 2014

(c) Springer Science + Business Media New York 2014

\begin{abstract}
Idiopathic epilepsies are thought to be almost entirely the result of genetic determinants, most of which remain undiscovered. It was once widely accepted that mutations that change the amino acid sequence of ion channel proteins were the major cause of these epilepsies, making epilepsy in general a "channelopathy." Since then, more comprehensive analysis of ion channel-encoding genes has largely rejected this hypothesis for common forms of epilepsy. Here, we discuss some of the assumptions that led to the channelopathy hypothesis and propose ways to avoid similar mistakes going forward. We also offer insight into promising genetic candidates that we believe play a major role in susceptibility to common forms of idiopathic epilepsy.
\end{abstract}

Keywords Genetic epilepsies - Ion channels - Complex disease $\cdot$ Causative variants $\cdot$ Exome sequencing

R. L. Subaran · D. A. Greenberg ( $₫)$

Nationwide Children's Hospital Research Institute, Columbus, $\mathrm{OH}$, USA

e-mail: David.Greenberg@nationwidechildrens.org

R. L. Subaran

e-mail: ryan.subaran@nationwidechildrens.org

D. A. Greenberg

Department of Neuroscience, The Ohio State University, Columbus, OH, USA

D. A. Greenberg

Department of Mathematical Medicine, The Ohio State University, Columbus, OH, USA

\section{Introduction}

The term epilepsy describes heterogeneous group of paroxysmal disorders, thought to be the result of disturbances in neural networks [1]. Family studies indicate that most common forms of idiopathic epilepsy have a strong, and often determining, genetic component [2-6]. In the late 1990 s, a succession of studies of rare, monogenic forms of epilepsy pointed to mutations in ion channel-encoding genes (channel genes) as potential causes of epilepsy. This helped lead to the prevailing idea that epilepsies are fundamentally a disease of ion channel dysfunction, i.e., channelopathies [7-10]. Despite the plausibility of the channelopathy model of epilepsy (and the evidence supporting it for some severe but rare forms of seizure disorders), it is now clear that the channelopathy hypothesis is not sufficient to explain most of the susceptibility to common epilepsy [11]. In fact, channel gene mutations once thought to be absolutely causal for particular types of epilepsy have been shown to occur silently in healthy individuals [12••]. Whatever the contribution of channel gene mutations to epilepsy expression, it is likely more complex and less influential than previously believed.

The channelopathy hypothesis has led to valuable insight into some rare forms of epilepsy and into the basic neurophysiology underlying certain types of seizures. However, the certainty with which it was accepted hampered the search for causes of common epilepsy. In this review, we consider some of the lessons that can be learned from understanding the development of the channelopathy hypothesis. In addition, we address the events and paradigms that led the field of epilepsy genetics to focus nearly entirely on this (ultimately) overly optimistic model of epileptogenesis. Calling on our growing understanding of the structure of the human genome, we discuss ideas on 
how to avoid some of the pitfalls of the channelopathy era in current and future studies. Additionally, we point to what we feel to be some of the success stories in the search for common epilepsy genes.

\section{Some Key Assumptions...}

Each approach to gene discovery makes certain assumptions about disease model such as, for example, frequency of disease alleles [13]. Through the course of its development, the channelopathy hypothesis made two assumptions that more recent research has shown to be flawed.

Assumption \#1 Dominant, predicted loss-of-function mutations are virtually absent in healthy individuals.

During the development of the channelopathy hypothesis, several studies reported causative mutations found through sequencing of known channel genes in patients, often in a region of interest (e.g., a linkage peak), and identifying variants predicted to affect gene function. These specific variants, and only those, would then be examined in healthy controls and, if found absent, would be deemed to be the cause of the epilepsy. In order for this approach to actually identify valid causative genes, one must assume that the probability of finding putative lossof-function mutations in any given gene is normally quite low. If the probability of finding any rare variant in a given gene is higher than anticipated, the assumption is violated and the results of studies starting from such assumptions must be reconsidered. We now know that rare, predicted loss-of-function mutations are, in fact, far more common than once thought, even in healthy individuals [14••]. Therefore, finding such a mutation segregating in a region of linkage but absent in controls is a limited indicator. Instead, the distribution of all mutations in the gene must be considered in comparisons between cases and controls in order to minimize type I error [15]. For the studies that led to support of the channelopathy hypothesis of epilepsy, the overall frequency of mutations in whatever channel gene was being studied was usually not mentioned. It is, therefore, likely that the inflated type I error for those studies can actually be quite high.

Assumption \#2 Our existing knowledge of physiology helps us to know which genes to prioritize.

As early as the 1950s, the effect of ion channels on neuronal excitability had been described in model systems. Seizure-like phenotypes such as the Drosophila "shaker" phenotype [16] are caused by mutations in channel genes. It is easy to imagine how mutations in homologous genes in humans can lead to increased susceptibility to seizure disorders. With their known well-characterized role in neuronal excitability, channel genes were high-priority candidate genes during the development of the channelopathy hypothesis. However, this hypothesis largely oversimplifies the complex nature of neuronal excitability regulation, a process involving many cellular factors and activities, the disruption of any of which factors could conceivably result in seizures. Work in model organisms where genetic networks have been thoroughly dissected show that for a given phenotype, causative mutations often lie in any of a wide variety of signaling factors such as kinases and transcription factors as well as their regulators, effectors, and targets, or even in genes involved in simple metabolic and housekeeping processes. Many of these genes were not a priori intuitive candidates for the phenotype of interest. The focus on channel genes as the only candidates to study in finding variants involved in epilepsy only considers a small aspect of the totality of control in neuronal circuitry. Moreover, a candidate gene approach inherently limits the discovery of disease genes to those that have been previously (or imagined to be) connected to a phenotype, thus precluding the elucidation of novel aspects of the molecular biology behind disease.

Examination of some of the initial studies implicating channel genes in epilepsy illustrates how the assumptions outlined above do indeed increase type I error. It also highlights the difficulty and ambiguity that comes with assigning causality in genetic human disease. Therefore, as we discuss next, several aspects of these initial channel gene studies bear revisiting.

\section{History of the Channelopathy Hypothesis}

\section{CHRNA4, KCQN2/3, Both, or Neither?}

Some of the earliest evidence supporting the notion of epilepsy as a channelopathy came from studies of two separate channels, the neural acetylcholine nicotinic receptor (nAChR) and the $\mathrm{K}_{\mathrm{v}} 7$ potassium "shaker" channel (KQT). Each was thought to be involved in distinct, rare, highly penetrant forms of epilepsy; namely, autosomal dominant nocturnal frontal lobe epilepsy (ADNFLE) and benign familial neonatal seizures (BFNS). Initially, linkage to BFNS was reported on chromosomes 20q13 [17, 18] and $8 q 24$ [19]. The 20q13 region (which has also been implicated in a specific electroencephalogram (EEG) phenotype [20] ) was found to contain the gene encoding the nAChR a4 subunit, CHRNA4 [21]. It can easily be imagined how disrupting the signal involving the neurotransmitter acetylcholine might lead to a seizure disorder like BFNS, thus making the nAChR subunit CHRNA4 an intuitive candidate gene. To investigate whether or not mutations in the nAChR subunit CHRNA4 were the source of the linkage 
signal observed on 20q13, the CHRNA4 exons were sequenced in $1210 \mathrm{q}$-linked BFNS families. One of these 12 families was found to have a nonsense mutation, which segregated completely with disease [22]. Therefore, a severe mutation in a strong candidate gene was shown to segregate with an epilepsy phenotype, providing some of the first evidence that tied a human, genetic epilepsy to a channel gene. Soon after, a CHRNA4 missense mutation in a conserved residue was found to segregate with another rare Mendelian epilepsy, autosomal dominant nocturnal frontal lobe epilepsy (ADNFLE), in a large family that showed linkage to the same region. The mutation was not found in 333 healthy controls [23, 24]. This was followed by the discovery of an insertion in the nAChR subunit CHRNA4 in a separate ADNFLE pedigree [25]. Thus, evidence seemed to be mounting for a role for CHRNA4 in epilepsy susceptibility. The data suggested a pleiotropic effect of the gene on at least two different epilepsy phenotypes: one, BFNS, characterized mainly by generalized seizures, the other, ADNFLE, by focal seizures. However, in 1998, two separate reports focusing on the KQT channel suggested that an entirely different gene was the cause of $20 \mathrm{q}$-linked BFNS. The first study found six of 12, 20qlinked BFNS families harbored mutations in the KQT gene, $K C N Q 2$, including two frameshifts, two missense, one deletion, and one splice-site mutation. These mutations all segregated completely with disease in the families where they were identified and were not found in controls [26, 27]. The second study identified a $K C N Q 2$ truncation in a large BFNS family [28]. So, which chr20q gene, the nAChR channel gene CHRNA4 or the KQT channel gene, $K C Q N 2$ was the actual cause of chr20q-linked BFNS?

The two genes, CHRNA4 and KCQN2, are separated by only $60 \mathrm{~kb}$, so alleles of each will almost always cosegregate. In a family-based study, a separation that small makes it difficult to disentangle the effect of mutations found in either gene. One key piece of evidence in distinguishing which gene was the cause of BFNS came from the second suggested BFNS linkage locus. Recall that, in addition to chr20q, linkage to BFNS was also observed at $8 \mathrm{q} 24$ [19]. It is here that a missense mutation in a conserved residue of another KQT channel gene that is a homolog of $K C N Q 2, K C N Q 3$, was found to segregate with disease in a large 8q24-linked BFNS pedigree [29]. The fact that mutations in two KQT channel genes segregated in separate BFNS families helped establish the idea that BFNS is caused by defects in the KQT channel rather than the nAChR channel. Nonetheless, the difficulties of assigning causality to one gene or the other at chr20q for BFNS illustrates how the assumptions imposed on some of early channel gene studies can be fraught with peril. It seems intuitive that a nonsense mutation leading to a truncated CHRNA4 gene product must disrupt normal
$\mathrm{nAChR}$ signaling and if there is a disruption in $\mathrm{nAChR}$ signaling, it must be the cause of BFNS, especially with the mutation segregating with affected offspring. However, recall that Assumption 1, noted above, is that the frequency of loss-of-function mutations in healthy individuals is very low. We now know this is not necessarily true. Genome analysis has shown that a normal human genome can tolerate rare nonsense mutations in several genes, even in the homozygous state [14*0]. This might have been made clear had the entire nAChR subunit CHRNA4 gene been sequenced in controls in the initial study. In addition, recall that Assumption 2 is that the genes involved will be intuitive candidates. While CHRNA4 was an excellent intuitive candidate, it was simply not the cause of BFNS.

It is worth noting that although the connection of CHRNA4 to BFNS has been debunked, it is nonetheless accepted as a major cause of ADNFLE. Despite the fact that the KQT subunit $K C N Q 2$ and not the nAChR subunit CHRNA4 turned out to be the likely BFNS gene the role of CHRNA4 gained acceptance as the cause of ADNFLE in some families [30]. Mutations in a separate nAChR subunit, CHRNB2, have since been found segregating with ADNFLE in some large families [31, 32]. In one largescale study, microdeletions in a region of $15 \mathrm{q} 13.3$ spanning seven genes, including CHRNA7 (nAChR $\alpha 7$ subunit), were found in $1 \%$ of (12 out of 1,223) idiopathic generalized epilepsy cases [33] and not in controls. Therefore, there might be some role for the nAChR channel in seizure disorders, although importantly, not in the most common forms of epilepsy.

\section{$\mathrm{Na}_{\mathrm{v}} 1$ and $\mathrm{GABA}(\mathrm{A}) \mathrm{R}$}

During the development of the channelopathy hypothesis, the $\mathrm{Na}_{\mathrm{v}} 1$ sodium channel would also be implicated as a cause of human epilepsy. By the mid-1990s, a role for sodium channels in seizure disorders had been long suspected. In addition to being intriguing candidate genes for neurological disorders based on their known function in neurotransmission, $\mathrm{Na}^{+}$channel activity is known to be important in neurons in mouse models of spontaneous seizure disorders [34]. In 1998, a C121W substitution was reported [35] in a $\mathrm{Na}_{\mathrm{v}} 1$ regulatory $\beta 1$ subunit $(S C N 1 B$, chromosome $19 \mathrm{q} 13$ ) in a large GEFS + (generalized epilepsy with febrile seizures plus [36]) family that showed linkage to chr19q13. While the C121W mutation in SCN1B did not segregate in family members who had simple febrile seizures as the only phenotype, it was found to segregate with other forms of epilepsy, including in individuals with complex febrile seizures compounded with other phenotypes. However, certain affected individuals did not carry the mutation, suggesting that multiple epilepsy genes are likely segregating in the family. The 
probability that multiple genes are each independently involved in seizure expression are segregating in a family is increased if families with a high proportion of affected individuals are preferentially ascertained [37]. After the $S C N 1 B$ finding for GEFS + , a separate study identified two separate missense mutations in another $\mathrm{Na}_{\mathrm{v}} 1$ subunit, (the a1 subunit-encoding SCNIA gene on chromosome 2q), in two different GEFS + families showing linkage to chr2q [38]. Therefore, similar to the KQT channel for BFNS, mutations in two genes for the same channel-in this case the $\mathrm{Na}_{\mathrm{v}} 1$ channel and the $S C N 1 A$ and $S C N 1 B$ genes-were implicated in the same epilepsy "syndrome", in this case GEFS + . However, while phenotypes such as the KQTchannel disease BFNS and the nAChR-channel disease ADNFLE are quite rare, individuals in families with GEFS + (a family-based designation which is also quite rare) can exhibit symptoms similar to those seen in patients with common idiopathic epilepsies. This makes these genes appear to be more apt examples of what might be going on in complex forms of the disease. And so, the findings for $\mathrm{Na}_{\mathrm{v}} 1$ subunits in GEFS+ perhaps added the notions that the channelopathy hypothesis might be further generalizable beyond rare Mendelian forms of epilepsy.

$\mathrm{Na}_{\mathrm{v}} 1$ subunits, particularly the $\alpha$ subunit-encoding SCN1A gene, could provide some insight in explaining disease in individuals from GEFS+ families. It has been found that about $10 \%$ of examined families classified as GEFS+ segregate a variety of mutations in SCNIA [39]. However, the most important finding about SCN1A is its role in causing another rare, but far more devastating disease, severe myoclonic epilepsy of infancy (SMEI) or Dravet syndrome [40-43]. Roughly $80 \%$ of Dravet syndrome patients harbor some SCN1A variant, most often the result of a de novo mutation [44]. Sometimes, albeit rarely, a mutation in a Dravet's patient is inherited from an unaffected parent, which raises suspicions of parental mosaicism or a requirement for the presence of mutations in other genes for disease expression [45]. When one also notes that, even in Dravet cases shown to be due to de novo mutations, there is often a family history of febrile seizures (FS), it further suggests some contribution of additional risk factors $[39,46]$. Mutations in the $S C N 2 A$ gene have also been reported to lead to Dravet syndrome-like phenotypes [47, 48], as has a recessive $S C N 1 B$ mutation in a single case [49]. Because of its severe morbidity, genetic findings involving Dravet syndrome are particularly valuable to patients and family members in understanding disease. The effect of mutations in subunits of $\mathrm{Na}_{\mathrm{v}} 1$ on Dravet syndrome is one of the clearest cases of a channel gene affecting an epilepsy syndrome. There is also some evidence that other $\mathrm{Na}_{\mathrm{v}} 1$ subunit genes, SCN3A, SCN8A, and $S C N 9 A$, may also contribute to epilepsy in a few cases [44]. However, as will prove to be the theme for most follow-up channel studies, mutations in $\mathrm{Na}_{\mathrm{v}} 1$ subunits do not seem to be relevant in most other common forms of epilepsy.

It is worth noting that, similar to $K C N Q 2$ and CHRNA4, there are also questions related to the proximity of other genes to $\mathrm{Na}^{+}$channel genes that must be considered when addressing the identification of causal variants. For example, the $\mathrm{Na}_{\mathrm{v}} 1 \alpha$ subunit gene cluster is implicated in various forms of epilepsy. This cluster contains the SCN1A, $S N C 2 A, S C N 3 A, S C N 7 A$, and SCN9A genes, all within a region less than $1.3 \mathrm{Mb}$. Other proximity issues might be less obvious yet even more dramatic. The LGI4 gene, recently implicated in forms of CAE and BFNS [50, 51], lies only $84 \mathrm{~kb}$ from the $S C N 1 B$ gene, reported causative in multiple epilepsies (see above). In each case, the possibility that segregation of a mutation in one gene might simply be a consequence of its proximity to the causal gene in the cluster must be seriously considered.

\section{Follow-Up Channel Gene Studies}

The discoveries regarding the potential effects of channel genes on Mendelian epilepsies have paved the way for increasing numbers of epilepsy genetic studies focused on channel and channel-related genes. Not only have followup studies prioritized these specific genes as strong candidates, but they have also turned to other subunits of their cognate channel complexes. Some studies have even turned to completely different kinds of ion channels, such as the $\gamma$-aminobutyric acid (GABA) receptor and the T-type $\mathrm{Ca}^{2+}$ channels, that had not yet been implicated in epilepsy [52, 53]. Some studies showed that these channels were, in fact, mutated in specific families, but these studies largely made the same assumptions that we have already discussed. Although many leads were generated through examining channel genes in epilepsy, the evidence for their involvement in any but the rare families in which they were found has been unconvincing. Overall, studies seeking to independently observe the effects reported for channel genes in new epilepsy samples failed to turn up any significant findings [54]. This called into question the true generalizability of the channel gene model of epilepsy etiology. This lack of reproducibility, along with the small effects observed in follow-up findings, suggests that simple mutations in channel genes could not explain the substantial heritable component attributed to idiopathic epilepsies [11]. Prima facie, this might not seem particularly surprising, indeed, most studies of common disease suffer from of this kind of observed "missing heritability" [55].

One reason for not observing the expected genetic contribution of a given gene is the involvement of multiple genes in disease expression. Such multiple-gene involvement can be allelic heterogeneity (i.e., there are multiple 
disease alleles at one locus, thus making finding association with any one of them difficult) or can be locus-based (i.e., the effect of disease-related allele(s) at loci in other parts of the genome) each independently producing disease with indistinguishable phenotypes. Multiple gene contributions can also be additive, in which a certain threshold, or count, of total disease-related alleles at many loci must be attained in order to achieve phenotypic expression [56]. Still another, similar, and not mutually exclusive, contribution can come from the influence of interacting alleles necessary for expression of a phenotype (i.e., epistasis) which evidence suggests underlie common forms of epilepsy [37, 54]. There is evidence that genetic interactions between channel gene mutations can influence phenotype. For example, SCN1A and SCN2A might combine to influence expression of febrile seizures and Dravet syndrome [46, 57] and might explain why even Dravet syndrome cases with de novo SCNIA mutations sometimes have a family history of FS. In addition, animal models suggest that, for example, Scn $2 a$ mutations in combination with Kcnq2 mutations can lead to more severe epilepsy phenotypes than either mutation alone [58]. Conversely, channel gene mutations can be compensatory. Specific Scn8a mutations, which can be harmful on their own, can overcome the viability defects exhibited by Scnla-deficient mice [59, 60]. In another such example, it has been shown that the separate seizure phenotypes caused by mutations in Kcnla (absence seizures) and Cacnala (limbic seizures) in mice can be mutually suppressed when both genes were mutated [61]. Recognition of gene-gene interactions is likely to be essential to understanding the influence of different genes on epilepsy expression and might explain why the effect of one particular channel gene can seem small when examined in isolation.

If the unidentified genetic contribution of channel genes to common epilepsy is attributable to factors such as epistatic interactions or genetic heterogeneity, then a comprehensive survey of channel gene variation across the genome might reveal effects that could be missed by examining only a single gene at a time or a small subset of channel genes. While any given channel gene mutation on its own might be a poor predictor of epilepsy susceptibility, it is possible that the overall channel gene mutational profile (or burden) in an individual might be a more powerful indicator of disease risk [12••, 62, 63]. In the same way that certain kinds of cancers can be the result of multiple hits in tumorigenic pathways [64], idiopathic epilepsies might also be the manifestation of exceeding some "hit" threshold [56], possibly in channel genes.

To measure the variation in channel genes as a class of genes and assess the effect on epilepsy, Klassen et al. [12••] Sanger-sequenced the exons of 237 (out of over 400) channel genes (including genes and their orthologs) previously purported to be involved in epilepsy in 152 idiopathic epilepsy cases and 139 matched controls. The analysis revealed a total of 668 missense mutations, thus proving false the assumption that coding mutations in channel genes are rare events.

Under multiple forms of analysis, the data generated by Klassen et al. suggest a trend toward an enrichment of channel gene mutations in cases. However, these apparent trends do not meet statistical significance when tested. Of the 668 missense mutations found in the dataset, 368 were found only in cases, while 78 were found only in controls, and 222 were seen in both. When they examine the rare missense mutations (substitutions) predicted to have the most severe effect on the protein, the observed effects also did not reach statistical significance. Nonsense mutations generally need no estimator of their likely effect on gene products. They lead to truncations, thus making their effect on function more straightforward to predict than most other mutations. When nonsense mutations in channel genes were examined, eight were found only in cases, while one was found only in controls, with no overlap. Four nonsense mutations and eight splice-site variants were found to be rare (frequency $<0.05$ in the dataset). This group of 12 likely severe variants were tested for association with epilepsy, but the distribution of these variants among cases and controls did not differ significantly [false discovery rate (FDR) of 0.11 under the null].

There were also 43 missense mutations in genes reported to cause human epilepsies found. While 20 of these reported human epilepsy mutations were indeed found exclusively among cases, five were found exclusively in controls including SCNIA R393H mutation, presumed to be causative for Dravet syndrome [40].

Of all purported human epilepsy channel genes as a whole, at least one substitution was found in $96.1 \%$ of cases and $66.9 \%$ of controls. As we have pointed out, many of the initial channel gene studies failed to report overall frequency of coding mutations of the channel controls. If this frequency is close to the $66.9 \%$ reported for these genes by Klassen, et al., type I error in these studies was almost certainly underestimated. Furthermore, knowing that healthy individuals can harbor variants reported as being causal has huge implications for how these mutations should be interpreted in the clinic and in genetic testing and counseling.

Klassen, et al. also examined the overall mutational load of reported human epilepsy channel genes in order to evaluate a multi-hit or threshold model of epileptogenesis. They found that $77.6 \%$ of cases have mutations in multiple purported human epilepsy genes, compared to only $29.5 \%$ in controls. While it is obvious that this particular test would yield a statistically significant association (this gives a simple $2 \times 2$ Pearson's $\chi^{2}(1 \mathrm{DF})$ value of 67.0 with an 
odds ration of 8.30 (95\%CI 4.90-14.06)), the data show that even at this comprehensive scale, the channel gene profile still does not completely explain total risk. Moreover, while the highest burden of epilepsy channel gene mutations observed in cases was nine, the highest in controls was seven. This means that even healthy individuals can have a high burden of putatively pathogenic channel gene mutations. The channel gene profile is also far from predictive.

While the relatively small sample size of this study might yield insufficient power to detect significant effects and the phenotypic characterizations are somewhat unclear, overall, the data suggest that although the interaction is not simple (and the prediction of disease is not straightforward), channel genes might indeed play some role in susceptibility to epilepsy. Even when considering multiple hits in human epilepsy genes it is clear that any given healthy individual might still carry a substantial load of the putative channel gene mutational burden. Issues of genetic noise, in the absence of a "healthy" reference genome, can complicate individualized disease prediction [12••]. Klassen, et al. concludes that an emphasis on channel gene mutation configuration rather than mere load is necessary for prediction of disease. Much of the evidence to date suggests that while channel genes may contribute to underlying susceptibility, perhaps they are not the most important genes conferring susceptibility to common epilepsy.

\section{Moving Forward}

Given some of the issues surrounding the approaches that led to the channelopathy hypothesis and its failure to explain susceptibility to most forms of epilepsy, other paradigms of epileptogenesis must be considered.

\section{Genome-Wide Association Studies of Common Epilepsy}

Genome-wide analyses (e.g., linkage or association analyses based on genome-wide marker scans) have been an important tool for finding genetic effects without making assumptions regarding the identity or class of gene involved. These approaches generally do not place priors on any given region of the genome, but rather treat each genomic locus equally a priori. In genome-wide association studies (GWAS) one uses markers, usually single-nucleotide polymorphisms, throughout the genome to test whether any of them are associated with the disease, i.e., in linkage disequilibrium with the disease allele at a disease locus. However, although this approach makes no assumptions regarding the class of gene involved, it does makes the assumption that individuals that share common phenotypes do so due to the presence of common inherited ancestral alleles [55]. The more this assumption is violated, i.e., the greater the number of disease-causing alleles in the population that can lead to a common phenotype, the more diminished is the ability to find association [65]. Even if mutations in a single gene are completely responsible for disease, if each mutation in the gene is individually rare, there is virtually no power to detect a genetic effect using association. Furthermore, even under conditions conducive to finding genetic effects by association, often prohibitively large numbers of cases and controls are required to detect effects in GWAS samples, a situation exacerbated by the need to correct for multiple genome-wide tests [66]. Complications such as these are likely the reason that GWAS findings for common forms of epilepsies have been limited. One GWAS on idiopathic epilepsy did show evidence for association at four separate loci $(1 \mathrm{q} 43,2 \mathrm{p} 16.1$, $2 \mathrm{q} 22.3$, and $17 \mathrm{q} 21.32$ ). However, as has been the pattern in other diseases, the results suggested that the alleles discovered accounted for a very small portion of the relative risk [67]. (Of note, none of the genes of interest mentioned were channel genes.) In a separate study that identified 87,255 functional (missense, nonsense, or splice-site) variants by exome sequencing of idiopathic epilepsy cases and controls, no single variant showed significant evidence of association [68•]. Given the lack of strong findings from GWAS for common epilepsies it is reasonable to conclude that the "common disease, common allele" hypothesis cannot explain the majority of risk for epilepsy susceptibility and that approaches that do not impose this assumption must be considered.

\section{Other Studies}

While mutations that cause epilepsy may not necessarily occur in channel genes, they might still affect channel gene function. For example, loss-of-function mutations in the non-channel gene LGII were shown to cause autosomal dominant partial epilepsy with auditory features (ADPEAF) [69]. LGII itself is not a channel gene; rather it encodes a secreted synaptic protein that associates with a $\mathrm{K}_{\mathrm{v}} 1.1$ potassium channel subunit and regulates channel activity. LGII mutations that cause ADPEAF appear to work by compromising this regulatory function [70]. However, LGII is not itself a channel gene and would not have been identified by sequencing candidate channel genes. The effect of LGI1 might also occur during neural development (a process we will discuss next in the context of epilepsy), as mouse studies show, it is highly expressed in the developing brain [71].

Several studies have published strong linkage signals for relatively large samples of families with more common forms of epilepsy [72-77]. It is almost certain that there are genes with large effects for common epilepsies. Many of 
these genes might have little to do with channel function directly. For instance, the ELP4-PAX6 locus, which has no known direct connection to ion channel function, shows strong genetic linkage $(\mathrm{LOD}=4.5)$ and association ( $p$ value $=0.0002)$ to the EEG trait of centro-temporal spikes seen in rolandic epilepsy families [77] and pleiotropy with verbal dyspraxia in RE families $(\mathrm{HLOD}=7.5$ ) [78]. In addition to such examples, there is evidence that defects in specific pathways can have a significant role on seizure disorder phenotype.

\section{Neural Development}

Events during neural development are also important in later neuropsychiatric outcomes. It has long been established that even extremely small changes during neural development can lead to drastic effects on phenotypes. In epilepsy, it has been demonstrated in animal models that the epileptogenic effects of the GEFS + GABRG2 R43Q and the ADNFLE CHRNB2 V287L alleles occur mainly during key windows in development $[79,80]$. Some genes that govern developmental processes in neurons have been implicated in epilepsy, including common forms with complex inheritance. For example, EFHCl is a putative epilepsy gene with no obvious role in ion channel function. EFHCl encodes a $\mathrm{Ca}^{2+}$-binding microtubule-associated protein involved in neuronal proliferation and is expressed in the developing and adult mammalian brain [81, 82•]. Strong linkage to the region was identified [83] and several mutations in EFHCl have been identified in a subset of JME families, mostly of Mexican and Central American ancestry [76]. Subsequent studies have also shown that mice deficient in $E F H C 1$ show increased susceptibility to spontaneous seizures [84] bridging a neuronal developmental phenotype to seizure outcomes for this gene.

Another connection between neural development and a common idiopathic epilepsy comes from $B R D 2$, which is genetically linked to JME (LOD $=4.2$ ) and to the epileptiform EEGs seen interictally both in JME patients and in some family members without seizures [72, 73, 85]. BRD2 on chromosome $6 \mathrm{p} 21$ was the first identified locus for a common epilepsy. It has also been linked to, and associated with EEG photosensitivity $[86,87]$ and specific alleles of $B R D 2$ have been associated with both JME and in photoconvulsive EEG [75, 87]. However, in contrast to EFHC1, which was first identified in families reporting Mexican and Central American ancestry, the evidence for $B R D 2$ seems to be specific to Caucasians. Association studies $[75,86,88,89]$ have supported the importance of $B R D 2$ in JME in Caucasians, making it one of the most replicated findings in epilepsy genetics. A difficulty in understanding how $B R D 2$ may lead to epilepsy is that its function is unknown. Studies in model systems have shown that $B R D 2$ is important in cell division and neural development [90, 91]. Heterozygous $B r d 2 \pm$ mice display increased seizure susceptibility to chemically induced seizures, and also exhibit both spontaneous seizures and abnormal EEGs [92•]. Heterozygous $B r d 2 \pm$ mice show a profound (up to $50 \%$ ) deficit of some types of GABAergic neurons, with a concomitant decrease in GAD67 (the ratelimiting GABA-synthesizing enzyme) expression, in brain regions directly implicated in seizure control $[92 \cdot, 93]$. This suggests that an important underlying factor for epilepsy susceptibility in JME is a regional deficit of inhibitory neurons. Such a deficit may arise during neural development and as $B R D 2$ is highly expressed in the developing nervous system before neural tube closure; total absence of $B r d 2$ in mice severely compromises CNS development in embryos, which do not survive developmental day 12 [91].

\section{Protein Trafficking}

Another example of a pathway that could play an important role in seizure phenotypes is the protein trafficking pathway. Neurotransmission is dependent on neuronal plasma membrane proteins (including ion channels) that are processed (e.g., glycosylated) through the ER and Golgi before being trafficked to the cell surface. Defects in protein as well as vesicular trafficking can lead to seizure disorders. Many of the putatively epileptogenic ion channel alleles mentioned in this review have been shown to be defective in trafficking and glycosylation rather than channel activity per se [49, 94-102]. Defects of trafficking need not lie in the protein itself being trafficked, but can also be in the general trafficking machinery, as is demonstrated by mutations in the Golgi vesicle transport gene GOSR2 that cause a form of progressive myoclonus epilepsy with early ataxia [103]. Thus, there appears to be evidence that, by several mechanisms, defects in protein trafficking can lead to the neuronal dysfunction underlying some seizure disorders making the genes involved in protein trafficking of potential interest in the study of common epilepsy.

\section{Metabolism}

Metabolic pathways, particularly those involved in energy homeostasis, have been implicated in neuropsychological phenotypes including bipolar disorder, schizophrenia, and anxiety disorders [104-106]. The brain is the most energetically demanding organ in the body and glucose is its preferred fuel. Many processes in neurons, including regulation of membrane potential by ion pumps, are ATPdependent and important compounds, including neurotransmitters, require metabolic intermediates for synthesis. Extreme hypoglycemia can lead to seizures even in healthy individuals and defects in the electron transport chain (the main source of ATP in animals) can lead to seizure disorders 
[107]. On the other hand, a sustained ketogenic diet can protect against seizures [108] through what is likely a separate mechanism [109, 110]. The machinery involved in producing ATP has been tied to numerous neurological defects, including epilepsy. It is possible that, among other things, perturbation of this machinery taxes the energydemanding brain and/or disturbs some critical metabolic balances. For example, a spectrum of generalized epilepsy phenotypes can be caused by mutation of the GLUT1encoded glucose transporter [111-114, 115•], and myoclonic epilepsy and ragged fiber disease (MERRF) is caused by mitochondrial mutations [116, 117]. In each case, a model for disturbance of normal energy metabolism and energy homeostasis in epilepsy susceptibility is implicated.

\section{Conclusions}

While these examples of pathways that could be involved in epilepsy susceptibility illustrate the possible role of nonchannel gene factors in disease, focusing on any one of these pathways would likely lead to the same problems and biases we have outlined in the channelopathy hypothesis. The genetics of common forms of epilepsy remain a puzzle. The use of forward genetics (i.e., finding genes based on phenotype) as opposed to a candidate gene approach (which assumes that certain kinds of genes are involved a priori) will be a powerful tool in advancing our understanding of the molecular biology of seizure phenotypes. Advances in sequencing technologies are sure to facilitate variant discovery. Molecular pathway analysis will afford greater power to catalog the genes and molecular processes underlying different forms of epilepsy, including those forms most common in the population. In so doing, we are likely to find that the genes that govern epilepsy susceptibility will turn out to be exciting and unexpected.

Disclosure R Subaran and D Greenberg both declare no conflicts of interest.

Human and Animal Rights and Informed Consent This article does not contain any studies with human or animal subjects performed by any of the authors.

\section{References}

Papers of particular interest, published recently, have been highlighted as:

- Of importance

- Of major importance

1. Spencer SS. Neural networks in human epilepsy: evidence of and implications for treatment. Epilepsia. 2002;43(3):219-27.
2. Inouye E. Observations on forty twin index cases with chronic epilepsy and their co-twins. J Nerv Ment Dis. 1960;130:401-16.

3. Corey LA, et al. The occurrence of epilepsy and febrile seizures in Virginian and Norwegian twins. Neurology. 1991;41(9): 1433-6.

4. Marshall AG, Hutchinson EO, Honisett J. Heredity in common diseases. A retrospective survey of twins in a hospital population. Br Med J. 1962;1(5270):1-6.

5. Schiottz-Christensen E. Genetic factors in febrile convulsions. An investigation of 64 same-sexed twin pairs. Acta Neurol Scand. 1972;48(5):538-46.

6. Sillanpaa M, et al. Genetic factors in epileptic seizures: evidence from a large twin population. Acta Neurol Scand. 1991;84(6): 523-6.

7. Ptacek LJ. Channelopathies: ion channel disorders of muscle as a paradigm for paroxysmal disorders of the nervous system. Neuromuscul Disord. 1997;7(4):250-5.

8. Berkovic SF, Scheffer IE. Genetics of the epilepsies. Curr Opin Neurol. 1999;12(2):177-82.

9. Hirose S, et al. Molecular genetics of human familial epilepsy syndromes. Epilepsia. 2002;43(Suppl 9):21-5.

10. Mulley JC, et al. Channelopathies as a genetic cause of epilepsy. Curr Opin Neurol. 2003;16(2):171-6.

11. Heron SE, et al. Channelopathies in idiopathic epilepsy. Neurotherapeutics. 2007;4(2):295-304.

12. • Klassen T, et al. Exome sequencing of ion channel genes reveals complex profiles confounding personal risk assessment in epilepsy. Cell. 2011;145(7):1036-48. This exome survey of ion channel encoding genes shows no significant differences in the frequncy or in the severity of ion channel mutations between idiopathic epielpsy patients and healthy controls and shows that healthy controls can harbor putatively pathogenic alleles.

13. Lupski JR, et al. Clan genomics and the complex architecture of human disease. Cell. 2011;147(1):32-43.

14. $\bullet$ Pelak K, et al. The characterization of twenty sequenced human genomes. PLoS Genet. 2010;6(9):e1001111. This shows by large-scale sequencing that the typical genome of healthy individuals can tolerate a surprisingly high number of mutations predicted to abolish the function of gene products.

15. Li B, Leal SM. Discovery of rare variants via sequencing: implications for the design of complex trait association studies. PLoS Genet. 2009;5(5):e1000481.

16. Salkoff L, Wyman R. Genetic modification of potassium channels in Drosophila Shaker mutants. Nature. 1981;293(5829):228-30.

17. Leppert $\mathrm{M}$, et al. Benign familial neonatal convulsions linked to genetic markers on chromosome 20. Nature. 1989;337(6208):647-8.

18. Malafosse A, et al. Confirmation of linkage of benign familial neonatal convulsions to D20S19 and D20S20. Hum Genet. 1992;89(1):54-8.

19. Lewis TB, et al. Genetic heterogeneity in benign familial neonatal convulsions: identification of a new locus on chromosome 8q. Am J Hum Genet. 1993;53(3):670-5.

20. Steinlein O, et al. Localization of a gene for the human lowvoltage EEG on $20 \mathrm{q}$ and genetic heterogeneity. Genomics. 1992;12(1):69-73.

21. Steinlein O, et al. Refinement of the localization of the gene for neuronal nicotinic acetylcholine receptor alpha 4 subunit (CHRNA4) to human chromosome 20q13.2-q13.3. Genomics. 1994;22(2):493-5.

22. Beck C, et al. A nonsense mutation in the alpha4 subunit of the nicotinic acetylcholine receptor (CHRNA4) cosegregates with 20q-linked benign neonatal familial convulsions (EBNI). Neurobiol Dis. 1994;1(1-2):95-9.

23. Phillips HA, et al. Localization of a gene for autosomal dominant nocturnal frontal lobe epilepsy to chromosome $20 \mathrm{q} 13.2$. Nat Genet. 1995;10(1):117-8. 
24. Steinlein OK, et al. A missense mutation in the neuronal nicotinic acetylcholine receptor alpha 4 subunit is associated with autosomal dominant nocturnal frontal lobe epilepsy. Nat Genet. 1995;11(2):201-3.

25. Steinlein OK, et al. An insertion mutation of the CHRNA4 gene in a family with autosomal dominant nocturnal frontal lobe epilepsy. Hum Mol Genet. 1997;6(6):943-7.

26. Leppert M, et al. Searching for human epilepsy genes: a progress report. Brain Pathol. 1993;3(4):357-69.

27. Singh NA, et al. A novel potassium channel gene, KCNQ2, is mutated in an inherited epilepsy of newborns. Nat Genet. 1998;18(1):25-9.

28. Biervert $\mathrm{C}$, et al. A potassium channel mutation in neonatal human epilepsy. Science. 1998;279(5349):403-6.

29. Charlier C, et al. A pore mutation in a novel KQT-like potassium channel gene in an idiopathic epilepsy family. Nat Genet. 1998;18(1):53-5.

30. Combi R, et al. Autosomal dominant nocturnal frontal lobe epilepsy-a critical overview. J Neurol. 2004;251(8):923-34.

31. Phillips HA, et al. CHRNB2 is the second acetylcholine receptor subunit associated with autosomal dominant nocturnal frontal lobe epilepsy. Am J Hum Genet. 2001;68(1):225-31.

32. Diaz-Otero F, et al. Autosomal dominant nocturnal frontal lobe epilepsy with a mutation in the CHRNB2 gene. Epilepsia. 2008;49(3):516-20.

33. Helbig I, et al. $15 \mathrm{q} 13.3$ microdeletions increase risk of idiopathic generalized epilepsy. Nat Genet. 2009;41(2):160-2.

34. Willow M, et al. Down regulation of sodium channels in nerve terminals of spontaneously epileptic mice. Cell Mol Neurobiol. 1986;6(2):213-20.

35. Wallace RH, et al. Febrile seizures and generalized epilepsy associated with a mutation in the $\mathrm{Na}+$-channel betal subunit gene SCN1B. Nat Genet. 1998;19(4):366-70.

36. Scheffer IE, Berkovic SF. Generalized epilepsy with febrile seizures plus. A genetic disorder with heterogeneous clinical phenotypes. Brain. 1997;120(Pt 3):479-90.

37. Greenberg DA, Durner M, Delgado-Escueta AV. Evidence for multiple gene loci in the expression of the common generalized epilepsies. Neurology. 1992;42(4 Suppl 5):56-62.

38. Escayg A, et al. Mutations of SCN1A, encoding a neuronal sodium channel, in two families with GEFS+2. Nat Genet. 2000;24(4):343-5.

39. Scheffer IE, et al. Dravet syndrome or genetic (generalized) epilepsy with febrile seizures plus? Brain Dev. 2009;31(5):394400.

40. Claes L, et al. De novo mutations in the sodium-channel gene SCN1A cause severe myoclonic epilepsy of infancy. Am J Hum Genet. 2001;68(6):1327-32.

41. Sugawara T, et al. Frequent mutations of SCN1A in severe myoclonic epilepsy in infancy. Neurology. 2002;58(7):1122-4.

42. Fujiwara $T$, et al. Mutations of sodium channel alpha subunit type 1 (SCN1A) in intractable childhood epilepsies with frequent generalized tonic-clonic seizures. Brain. 2003;126(Pt 3):531-46

43. Claes L, et al. De novo SCN1A mutations are a major cause of severe myoclonic epilepsy of infancy. Hum Mutat. 2003;21(6): 615-21.

44. Meisler MH, O'Brien JE, Sharkey LM. Sodium channel gene family: epilepsy mutations, gene interactions and modifier effects. J Physiol. 2010;588(Pt 11):1841-8.

45. Depienne $\mathrm{C}$, et al. Mechanisms for variable expressivity of inherited SCN1A mutations causing Dravet syndrome. J Med Genet. 2010;47(6):404-10.

46. Singh NA, et al. A role of SCN9A in human epilepsies, as a cause of febrile seizures and as a potential modifier of Dravet syndrome. PLoS Genet. 2009;5(9):e1000649.
47. Kamiya K, et al. A nonsense mutation of the sodium channel gene SCN2A in a patient with intractable epilepsy and mental decline. J Neurosci. 2004;24(11):2690-8.

48. Shi $X$, et al. Missense mutation of the sodium channel gene SCN2A causes Dravet syndrome. Brain Dev. 2009;31(10): 758-62.

49. Patino GA, et al. A functional null mutation of SCN1B in a patient with Dravet syndrome. J Neurosci. 2009;29(34): 10764-78.

50. Gu W, et al. Genotypic association of exonic LGI4 polymorphisms and childhood absence epilepsy. Neurogenetics. 2004;5(1):41-4.

51. Ishii A, et al. Positive association between benign familial infantile convulsions and LGI4. Brain Dev. 2010;32(7):538-43.

52. Helbig I, et al. Navigating the channels and beyond: unravelling the genetics of the epilepsies. Lancet Neurol. 2008;7(3):231-45.

53. Reid CA, Berkovic SF, Petrou S. Mechanisms of human inherited epilepsies. Prog Neurobiol. 2009;87(1):41-57.

54. Greenberg DA, Subaran R. Blinders, phenotype, and fashionable genetic analysis: a critical examination of the current state of epilepsy genetic studies. Epilepsia. 2011;52(1):1-9.

55. Manolio TA, et al. Finding the missing heritability of complex diseases. Nature. 2009;461(7265):747-53.

56. Falconer DS. Introduction to quantitative genetics. 2nd ed. London/New York: Longmans Green; 1981.

57. Peiffer A, et al. A locus for febrile seizures (FEB3) maps to chromosome 2q23-24. Ann Neurol. 1999;46(4):671-8.

58. Kearney JA, et al. Severe epilepsy resulting from genetic interaction between $\mathrm{Scn} 2 \mathrm{a}$ and Kcnq2. Hum Mol Genet. 2006;15(6):1043-8.

59. Martin MS, et al. The voltage-gated sodium channel Scn8a is a genetic modifier of severe myoclonic epilepsy of infancy. Hum Mol Genet. 2007;16(23):2892-9.

60. Hawkins NA, et al. Neuronal voltage-gated ion channels are genetic modifiers of generalized epilepsy with febrile seizures plus. Neurobiol Dis. 2011;41(3):655-60.

61. Glasscock E, et al. Masking epilepsy by combining two epilepsy genes. Nat Neurosci. 2007;10(12):1554-8.

62. Fraser FC. The multifactorial/threshold concept-uses and misuses. Teratology. 1976;14(3):267-80.

63. Wray NR, Goddard ME. Multi-locus models of genetic risk of disease. Genome Med. 2010;2(2):10.

64. Knudson AG Jr. Mutation and cancer: statistical study of retinoblastoma. Proc Natl Acad Sci USA. 1971;68(4):820-3.

65. Rodriguez-Murillo L, Greenberg DA. Genetic association analysis: a primer on how it works, its strengths and its weaknesses. Int J Androl. 2008;31(6):546-56.

66. Marjoram P, Zubair A, Nuzhdin SV. Post-GWAS: where next? More samples, more SNPs or more biology? Heredity (Edinb). 2014;112(1):79-88.

67. Steffens M, et al. Genome-wide association analysis of genetic generalized epilepsies implicates susceptibility loci at $1 \mathrm{q} 43$, 2p16.1, 2q22.3 and 17q21.32. Hum Mol Genet. 2012;21(24): $5359-72$.

68. Heinzen EL, et al. Exome sequencing followed by large-scale genotyping fails to identify single rare variants of large effect in idiopathic generalized epilepsy. Am J Hum Genet. 2012;91(2):293-302. This find no single SNP, exome-wide, to be associated with forms of epilepsy known to be caused largely by genetic factors.

69. Kalachikov S, et al. Mutations in LGI1 cause autosomal-dominant partial epilepsy with auditory features. Nat Genet. 2002;30(3):335-41.

70. Fukata Y, et al. Disruption of LGI1-linked synaptic complex causes abnormal synaptic transmission and epilepsy. Proc Natl Acad Sci USA. 2010;107(8):3799-804. 
71. Silva J, Wang G, Cowell JK. The temporal and spatial expression pattern of the LGI1 epilepsy predisposition gene during mouse embryonic cranial development. BMC Neurosci. 2011;12:43.

72. Greenberg DA, et al. Juvenile myoclonic epilepsy (JME) may be linked to the BF and HLA loci on human chromosome 6. Am J Med Genet. 1988;31(1):185-92.

73. Weissbecker KA, et al. Confirmation of linkage between juvenile myoclonic epilepsy locus and the HLA region of chromosome 6. Am J Med Genet. 1991;38(1):32-6.

74. Durner M, et al. Genome scan of idiopathic generalized epilepsy: evidence for major susceptibility gene and modifying genes influencing the seizure type. Ann Neurol. 2001;49(3):328-35.

75. Pal DK, et al. BRD2 (RING3) is a probable major susceptibility gene for common juvenile myoclonic epilepsy. Am J Hum Genet. 2003;73(2):261-70.

76. Suzuki T, et al. Mutations in EFHC1 cause juvenile myoclonic epilepsy. Nat Genet. 2004;36(8):842-9.

77. Strug LJ, et al. Centrotemporal sharp wave EEG trait in rolandic epilepsy maps to Elongator Protein Complex 4 (ELP4). Eur J Hum Genet. 2009;17(9):1171-81.

78. Pal DK, et al. Pleiotropic effects of the $11 \mathrm{p} 13$ locus on developmental verbal dyspraxia and EEG centrotemporal sharp waves. Genes Brain Behav. 2010;9(8):1004-12.

79. Chiu C, et al. Developmental impact of a familial GABAA receptor epilepsy mutation. Ann Neurol. 2008;64(3):284-93.

80. Manfredi I, et al. Expression of mutant beta2 nicotinic receptors during development is crucial for epileptogenesis. Hum Mol Genet. 2009;18(6): 1075-88.

81. de Nijs L, et al. EFHC1 interacts with microtubules to regulate cell division and cortical development. Nat Neurosci. 2009;12(10):1266-74.

82. - Leon C, et al. Distribution of EFHC1 or Myoclonin 1 in mouse neural structures. Epilepsy Res. 2010;88(2-3):196-207. This highlights possible cellular and molecular mechanisms by which the non-channel gene EFHC1 might influence susceptibility to common forms of epilepsy.

83. Liu AW, et al. Juvenile myoclonic epilepsy locus in chromosome 6p21.2-p11: linkage to convulsions and electroencephalography trait. Am J Hum Genet. 1995;57(2):368-81.

84. Suzuki T, et al. Efhc1 deficiency causes spontaneous myoclonus and increased seizure susceptibility. Hum Mol Genet. 2009; 18(6):1099-109.

85. Durner M, et al. Localization of idiopathic generalized epilepsy on chromosome $6 \mathrm{p}$ in families of juvenile myoclonic epilepsy patients. Neurology. 1991;41(10):1651-5.

86. Tauer U, et al. Genetic dissection of photosensitivity and its relation to idiopathic generalized epilepsy. Ann Neurol. 2005;57(6):866-73.

87. Lorenz S, et al. Association of BRD2 polymorphisms with photoparoxysmal response. Neurosci Lett. 2006;400(1-2):135-9.

88. Greenberg DA, et al. Reproducibility and complications in gene searches: linkage on chromosome 6 , heterogeneity, association, and maternal inheritance in juvenile myoclonic epilepsy. Am J Hum Genet. 2000;66(2):508-16.

89. Cavalleri GL, et al. A multicenter study of BRD2 as a risk factor for juvenile myoclonic epilepsy. Epilepsia. 2007;48(4):706-12.

90. Crowley T, et al. Change in nuclear-cytoplasmic localization of a double-bromodomain protein during proliferation and differentiation of mouse spinal cord and dorsal root ganglia. Brain Res Dev Brain Res. 2004;149(2):93-101.

91. Shang E, et al. Double bromodomain-containing gene Brd2 is essential for embryonic development in mouse. Dev Dyn. 2009;238(4):908-17.

92. - Velisek L, et al. GABAergic Neuron deficit as an idiopathic generalized epilepsy mechanism: the role of BRD2 haploinsufficiency in juvenile myoclonic epilepsy. PLoS One. 2011;6(8):e23656. This presents a model of how dysfunction of the non-channel gene BRD2 can lead to increased susceptibility to common epilepsy.

93. Shang E, et al. The bromodomain-containing gene BRD2 is regulated at transcription, splicing, and translation levels. J Cell Biochem. 2011;112(10):2784-93.

94. Kang JQ, Macdonald RL. The GABAA receptor gamma2 subunit R43Q mutation linked to childhood absence epilepsy and febrile seizures causes retention of alpha1beta2gamma2S receptors in the endoplasmic reticulum. J Neurosci. 2004;24(40):8672-7.

95. Kang JQ, Shen W, Macdonald RL. The GABRG2 mutation, Q351X, associated with generalized epilepsy with febrile seizures plus, has both loss of function and dominant-negative suppression. J Neurosci. 2009;29(9):2845-56.

96. Tanaka M, et al. Hyperglycosylation and reduced GABA currents of mutated GABRB3 polypeptide in remitting childhood absence epilepsy. Am J Hum Genet. 2008;82(6):1249-61.

97. Wen H, Levitan IB. Calmodulin is an auxiliary subunit of KCNQ2/ 3 potassium channels. J Neurosci. 2002;22(18):7991-8001.

98. Richards MC, et al. Novel mutations in the KCNQ2 gene link epilepsy to a dysfunction of the KCNQ2-calmodulin interaction. J Med Genet. 2004;41(3):e35.

99. Etxeberria A, et al. Calmodulin regulates the trafficking of KCNQ2 potassium channels. FASEB J. 2008;22(4):1135-43.

100. Rusconi R, et al. A rescuable folding defective Nav1.1 (SCN1A) sodium channel mutant causes GEFS+: common mechanism in Nav1.1 related epilepsies? Hum Mutat. 2009;30(7):E747-60.

101. Rusconi R, et al. Modulatory proteins can rescue a trafficking defective epileptogenic Nav1.1 Na+ channel mutant. J Neurosci. 2007;27(41):11037-46.

102. Saitsu $H$, et al. De novo mutations in the gene encoding STXBP1 (MUNC18-1) cause early infantile epileptic encephalopathy. Nat Genet. 2008;40(6):782-8.

103. Corbett MA, et al. A mutation in the Golgi Qb-SNARE gene GOSR2 causes progressive myoclonus epilepsy with early ataxia. Am J Hum Genet. 2011;88(5):657-63.

104. Yao JK, Reddy RD. Metabolic investigation in psychiatric disorders. Mol Neurobiol. 2005;31(1-3):193-203.

105. Masino SA, et al. Adenosine, ketogenic diet and epilepsy: the emerging therapeutic relationship between metabolism and brain activity. Curr Neuropharmacol. 2009;7(3):257-68.

106. Jou SH, Chiu NY, Liu CS. Mitochondrial dysfunction and psychiatric disorders. Chang Gung Med J. 2009;32(4):370-9.

107. El Sabbagh S, et al. Epileptic phenotypes in children with respiratory chain disorders. Epilepsia. 2010;51(7):1225-35.

108. Bough KJ, Rho JM. Anticonvulsant mechanisms of the ketogenic diet. Epilepsia. 2007;48(1):43-58.

109. Laschet JJ, et al. Glyceraldehyde-3-phosphate dehydrogenase is a GABAA receptor kinase linking glycolysis to neuronal inhibition. J Neurosci. 2004;24(35):7614-22.

110. Pumain R, et al. Lability of GABAA receptor function in human partial epilepsy: possible relationship to hypometabolism. Epilepsia. 2008;49(Suppl 8):87-90.

111. Weber YG, et al. GLUT1 mutations are a cause of paroxysmal exertion-induced dyskinesias and induce hemolytic anemia by a cation leak. J Clin Invest. 2008;118(6):2157-68.

112. Suls A, et al. Paroxysmal exercise-induced dyskinesia and epilepsy is due to mutations in SLC2A1, encoding the glucose transporter GLUT1. Brain. 2008;131(Pt 7):1831-44.

113. Schneider SA, et al. GLUT1 gene mutations cause sporadic paroxysmal exercise-induced dyskinesias. Mov Disord. 2009;24(11):1684-8.

114. Suls A, et al. Early-onset absence epilepsy caused by mutations in the glucose transporter GLUT1. Ann Neurol. 2009;66(3): 415-9. 
115. - Striano P, et al. GLUT1 mutations are a rare cause of familial idiopathic generalized epilepsy. Neurology. 2012;78(8):557-62. This shows that inherited defects in glucose metabolism can lead to increased seizure susceptibility.

116. Shoffner JM, et al. Myoclonic epilepsy and ragged-red fiber disease (MERRF) is associated with a mitochondrial DNA tRNA(Lys) mutation. Cell. 1990;61(6):931-7.
117. Canafoglia L, et al. Epileptic phenotypes associated with mitochondrial disorders. Neurology. 2001;56(10):1340-6. 